\title{
Observation of a Prominence Heating and the Heating Mechanism
}

\section{Ofman}

Hughes STX/NASA Goddard Space Flight Center, Code 682, Greenbelt, $M D$ 20771, USA

\section{Z. Mouradian}

Observatoire de Paris-Meudon, DASOP-CNRS URA 326, F-92195 Meudon Cedex, France

T. A. Kucera

SAC/NASA Goddard Space Flight Center, Code 682, Greenbelt, MD 20771, USA

\section{A. I. Poland}

NASA Goddard Space Flight Center, 682, Greenbelt, MD 20771, USA

\begin{abstract}
An arch-shaped prominence was observed with SUMERSOHO spectrometer on March 31-April 1, 1996. The observations were performed at three wavelengths in the Lyman continuum. Ten successive images were obtained at 41 minute time intervals. Based on the computed models of Gouttebroze-Heinzel-Vial we have determined the temperature distribution of the prominence using the intensity ratio of $876 \AA$ and 907 $\AA$. The observed time sequence shows that the prominence disappears by heating, i.e., ionization of hydrogen: a thermal DB. We model the heat input with the linearized MHD equations using a prescribed initial density and a broad-band spectrum of Alfvén waves and find a good qualitative agreement with observations. In the model the prominence is heated by the resonant absorption of Alfvén waves with frequencies that match the resonant condition for a particular flux tube structure that is determined by the magnetic field topology and density.
\end{abstract}

\section{Introduction}

We present the observations of an arch-shaped prominence obtained with the SUMER spectrometer on board the SOHO spacecraft on March 31-April 1, 1996. The observations were performed at three wavelengths in the Ly continuum. Ten successive images were obtained at 41 minute time intervals and show evidence of thermal sudden disappearances (DB) (see Mouradian et al. 1995). Based on computed models of Gouttebroze-Heinzel-Vial (GHV) (Gouttebroze, Heinzel, Vial 1993), we have determined the temperature distribution of the prominence 


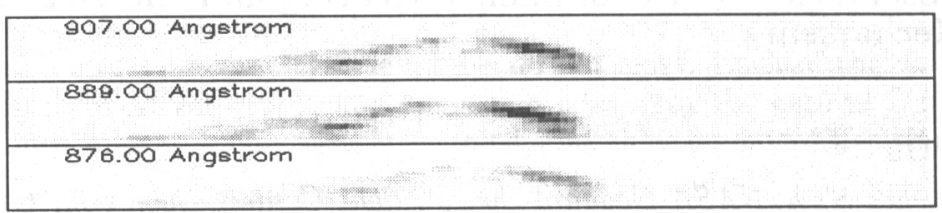

$1996-03-31720: 42: 19.035$

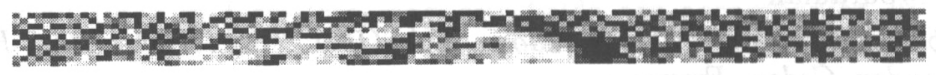

$<4300$.

9650

$>15000$

Temp. Kelvin

Figure 1. The image of the prominence in $876 \AA, 889 \AA$, and $907 \AA$ of Ly-continuum obtained by SUMER on March 31, 1996 at 20:42:19.035 UT (top three panels). The temperature distribution obtained from the ratio of $876 \AA$ and $907 \AA$ images and the GHV model (bottom).

using the intensity ratio of $876 \AA$ and $907 \AA$ images.

Resonant absorption of Alfvén waves was first suggested by Ionson (1978) as a nonthermal heating mechanism for coronal loops and since then has been extensively studied (see Ofman et al. 1995, and references therein). This mechanism appears to be a good candidate for prominence heating due to its short dissipation length and high efficiency in converting the wave energy to heat (Ofman and Mouradian 1996).

We compare the heating time scale obtained from SUMER observations to the resonant absorption heating model and find good qualitative agreement. However, a more realistic model that includes the effects of coupling between the prominence plasma and the chromosphere/photosphere is needed to explain the heating and cooling near the foot points.

\section{Observations}

An arch-shaped prominence was observed with the SOHO-SUMER spectrometer on March 31, 1996. Ten successive images in the Ly-continuum were obtained at 41 minutes with the resolution $1^{\prime \prime} \times 0.83^{\prime \prime} \approx 700 \times 600 \mathrm{~km}$. In Figure 1 we show the typical image of the prominence in $876 \AA, 889 \AA$, and $907 \AA$ of Ly-continuum taken on March 31, 1996 at 20:42:19.035 UT (top three panels). Based on the GHV model we have determined the temperature distribution of the prominence using the intensity ratio of $876 \AA$ and $907 \AA$ (lower panel). It is evident that regions of hotter and cooler material are concentrated near the footpoints with the hot material "on top" of the cool material above the limb.

The effects of heating and cooling are evident near the footpoints in regions of hot $(>15,000 \mathrm{~K})$ and cool $(\sim 4000-7000 \mathrm{~K})$ prominence material. In Figure 2 


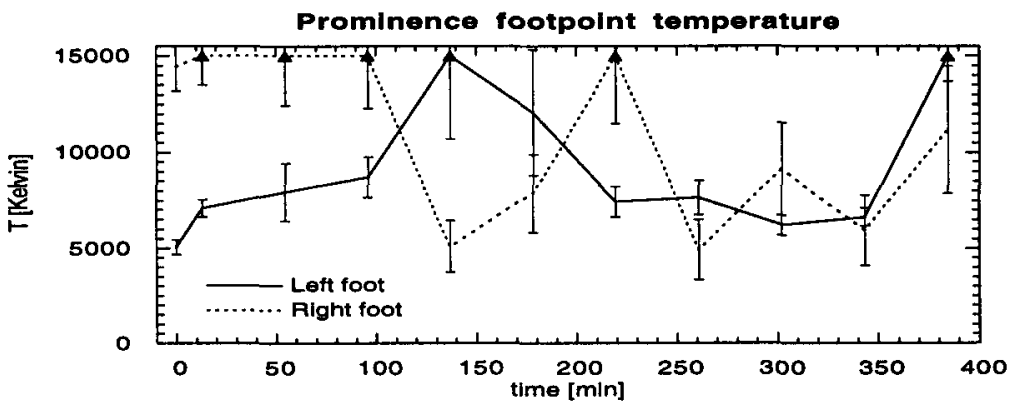

Figure 2. The time dependence of the temperature near the prominence footpoints determined from intensity ratio of $876 \AA$ and $907 \AA$ images obtained by SUMER on March 31-April 1, 1996. The arrows indicate temperatures above $15,000 \mathrm{~K}$ (outside the GHV model range).

we show the time dependence of the temperature near the prominence footpoints obtained by SUMER on March 31-April 1, 1996. The temperature error bars were calculated using the expression

$$
\frac{\Delta T}{T}=\sqrt{\frac{1}{I_{876}}+\frac{1}{I_{907}}},
$$

where $I_{876}$ and $I_{907}$ are the photon counts at the corresponding wavelengths, and we assumed Poisson statistics for the photons. Figure 2 was taken at particular points and shows cooling and heating. However, the observed time sequence of the full prominence image shows that parts of the prominence disappear by heating, i.e. ionization of hydrogen which is a thermal DB. The arrows indicate that temperatures above $15,000 \mathrm{~K}$ (outside the range of the GHV model) are present. The footpoint temperature varies on a timescale of 1-2 hours, which may indicate heating and cooling at this time scale. The temperature may also change due to flow of hotter or cooler material into the footpoints.

\section{Alfvén Wave Resonant Absorption Heating Model}

Here, we present a brief description of the Alfvén wave resonant absorption heating model for prominences. More details on the model can be found in Ofman and Mouradian (1996). We model a single flux tube (thread) of a coronal prominence by a slab of plasma (i.e., neglecting the effects of curvature) embedded in a uniform magnetic field. The $z$ direction is along the longitudinal magnetic field of the flux tube, the $y$ direction corresponds to the azimuthal direction of the flux tube, and the density inhomogeneity occurs in the $x$ direction, which corresponds to the radial direction of the flux tube.

The Alfvén waves are driven by a broad band (random) driving term in the momentum equation. In Figure 3 we show the temporal evolution of the heating rate obtained from the above model with $\tau_{A}=12.8 \mathrm{sec}$. It is evident that the heating varies in magnitude considerably due to the resonance effects of the 


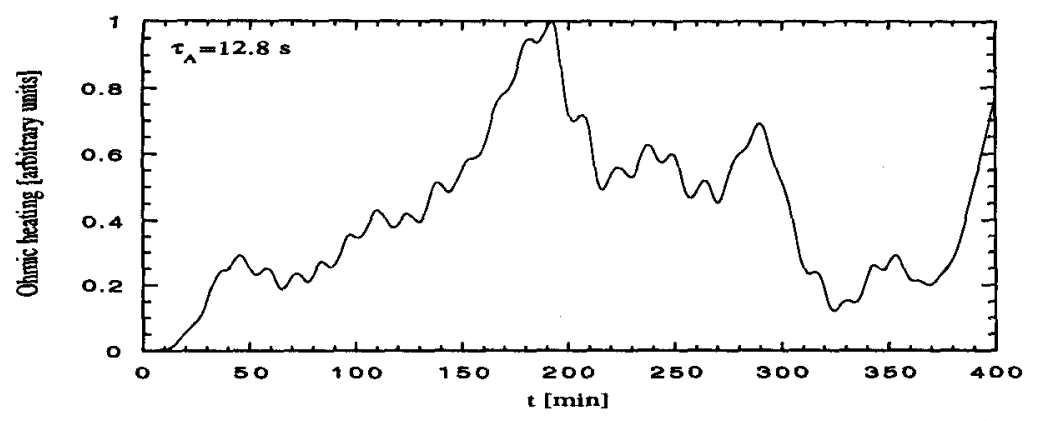

Figure 3. The temporal evolution of the heating rate in the resonant absorption model.

various frequencies in the time-dependent Alfvén wave power spectrum with the flux tube structure. When there is more power in the narrow band of frequencies near the global mode frequency of the flux tube - the heating increases. When the available power shifts to other frequencies - the heating decreases. The time scale for the decrease and increase in heating is determined by the geometry of the flux tube, the plasma parameters, and the supplied Alfvén wave spectrum. The evolution of the model heating is similar to the observed evolution of the footpoint temperature.

\section{Summary and Conclusions}

SUMER observations show that a quiescent prominence undergoes heating and cooling on time scales of 1-2 hours, and that the temperature distribution near the footpoints is different from the top of the prominence. Since the Alfven waves would be trapped and reflected differently at the footpoints and at the top of the prominence, we suggest that the Alfvén waves supply the necessary heat to the prominence.

We apply the resonant absorption heating model to prominences by solving the linear low- $\beta$ MHD equations and find that the heating time and global mode frequencies agree with the heating time and the wave frequencies observed in a typical quiescent prominence (Ofman and Mouradian 1996). We find that with a broad-band (random) Alfvén wave source the model prominence undergoes periods of high and low heat input, similar to the observed temperature evolution. However, chromospheric coupling, gravity and magnetic field geometry must be take into account to explain heating and cooling near the footpoints.

\section{References}

Gouttebroze, P., Heinzel, P. and Vial, J. C. 1993, A\&AS, 99, 513

Ionson, J. A. 1978, ApJ, 226, 650

Mouradian, Z., Soru-Escaut, I. and Pojoga, S. 1995, Solar Phys., 158, 269

Ofman, L., Davila, J.M. and Steinolfson, R.S. 1995, ApJ, 444, 471

Ofman, L. and Mouradian, Z. 1996, A\&A, 308, 631 